\title{
MULTI-FIELD THREE-NODE TRIANGULAR FINITE ELEMENT MODEL FOR HELMHOLTZ PROBLEM
}

\author{
K.Y.Sze ${ }^{1}$, Q.H.Zhang ${ }^{1,2}$, G.H.Liu ${ }^{3}$ \\ 1 Department of Mechanical Engineering, The University of Hong Kong, Pokfulam, Hong Kong, P.R.China. \\ 2 Department of Scientific Computing \& Computer Applications, Sun Yat-Sen University, Guangzhou, P.R.China. \\ kysze@hku.hk,zhangqh@hku.hk; flowce@hotmail.com
}

\begin{abstract}
In this paper, four three-node triangular finite element models which can readily be incorporated into the standard finite element program framework are devised via a multi-field variational functional for the bounded plane Helmholtz problem. In the models, boundary and domain fields are independently assumed. The former is constructed by nodal interpolation and the latter comprises non-singular solutions of the Helmholtz equation. The equality of the two fields are enforced along the element boundary. Among the four devised models, the most accurate one is $1 / 3$ to $1 / 2$ less erroneous than the conventional single-field model in most examples.
\end{abstract}

Keywords: Multi-field, hybrid, Trefftz, triangular, finite element, Helmholtz.

\section{Introduction}

A major challenge in finite element analysis of the Helmholtz problem is that the solution is spatially oscillating in the entire problem domain. While considerable computational saving can be realized by using graded meshes in stress analyses, the practice is not applicable to the Helmholtz problem. The high mesh-density requirement induces tremendous computing load when the wavenumber goes up. To better tackle the issue, a number of wave-based approaches that employ the non-singular solution sets for the Helmholtz equation have been proposed in the last decades. These include various Trefftz methods [1-11], the plane-wave basis method [12-15] and the discontinuous enrichment method [16-19], etc.

Among the Trefftz finite element methods, the least-square models [6,7], the traction-frame models [8] and the displacement-frame models [9-11] can be noted. All Trefftz finite element models possess their own domain modes which are extracted from a non-singular complete solution set. The least-square models are formulated by minimizing a weighted sum of the error norms of the boundary conditions and inter-element continuity conditions on the domain modes of the adjacent elements. "Displacement-frame" and "traction-frame" are inherited from Trefftz finite element formulation for elasticity problems $[8,20]$. The traction-frame (displacement-frame) models are equipped with boundary "traction" ("displacement") modes and the domain modes can be condensed at the element level. As these two categories of models possess two fields, i.e. the domain and boundary fields, they are also known as hybrid-Trefftz models. In Helmholtz problems, "displacement" and "traction" refer to the Helmholtz variable and its flux across the element boundary, respectively.

Published in Journal of Computational Acoustics, Vol.19, No.3 (2011) 317-334

(DOI: 10.1142/S0218396X11004353) 
In the plane-wave basis method, the plane-wave solutions are employed as the nodal enrichment functions in the context of the partition of unity finite element method [12-15]. The value of the Helmholtz variable at a node is the sum of the plane-wave modes propagating along different directions. Within the element, the Helmholtz variable is obtained by the conventional nodal interpolation. Thus, the system equation unknowns are the amplitudes of the plane-wave solutions at the nodes but not the nodal value of the Helmholtz variable.

In the discontinuous enrichment method, the coarse scale modes constructed by the nodal interpolation are enriched by plane-wave solutions. The enrichment which is intended to resolve the fine scale phenomenon induces discontinuity across the inter-element boundary [16-19]. Continuity is enforced by Lagrange multipliers. While the fine scale enrichment modes can be condensed at the element level, the multipliers which link the enrichments of adjacent elements enter the system equation.

Designing "small" multi-field or hybrid elements is indeed challenging. Here, "small" and "large" elements are those possessing respectively small and large numbers of nodal dofs which enter the global equation. In "large" elements, the nodal dofs are plenty and the elements are less sensitive to the choice and the number of domain modes. On the other hand, accuracy of the "small" element can vary significantly with respect to the domain modes and their number. Poorly chosen domain modes can even lead to rank deficiency as well as variance of the element matrix with respect to element translation, rotation and connectivity [21,22]. While the authors and their coworker have derived hybrid-Trefftz element models using plane-wave and Bessel solution sets for four-node quadrilateral, eight-node quadrilateral and six-node triangular elements [9-11], this paper will present four three-node triangular models. Again, the "displacement-frame" will be employed as the resulting elements can readily be plugged into the conventional finite element problem framework. In the first model, the domain modes are truncated from non-singular complete solution set formed by Bessel functions of the first kind. Hence, it is a hybrid-Trefftz model. In the second model, the zeroth order Bessel functions of the first kind, i.e. $J_{0}$, with different origins are employed as the domain modes. The idea resembles a meshless radial basis function (RBF) technique in which the radial basis functions among the Trefftz solution set are employed [23,24]. Note worthily, a mathematical proof on the completeness of the technique is pending. Both the third and fourth models employs plane-wave solutions in their domain fields. In most of the existing finite element formulations, the forward and backward plane-wave solutions are employed in pair, i.e. $\exp \left[i k r^{T} \varphi\right]$ and $\exp \left[i k r^{T}(-\varphi)\right]$ in which $\mathbf{r}=\{x, y\}^{T}$ is the position vector and $\varphi=\{\cos \varphi, \sin \varphi\}^{T}$ is unit vector along the propagation direction $\varphi$. Without changing the basis, the two solutions can be expressed as:

$$
\cos \left(k \mathbf{r}^{T} \boldsymbol{\varphi}\right) \text { and } \sin \left(k \mathbf{r}^{T} \boldsymbol{\varphi}\right) .
$$

However, the pairwise use of two solutions is not considered here as the number of node in the targeted element is three which is a small odd number. When two plane-wave modes along a single pair of directions are employed, the element is rank deficient. When four plane-wave modes along two pairs of directions are employed, the resulting three-node element is often less accurate than the conventional element in our computational trials. Furthermore, it is impossible to select two pairs of directions such that the element is invariant. It will be seen that only one of the two sinusoidal functions in Eq.(1) and locally defined origins of $(x, y)$ are employed to define the element domain field in the third and fourth models. 


\section{Conventional Formulation and Three-Node Triangle}

Helmholtz equation is often introduced by using steady-state acoustics or vibration of tensioned membrane. In the former, the Helmholtz variable $u$ can be the spatial amplitude of the pressure or the velocity potential. With the problem domain $\Omega$ partitioned into finite elements $\Omega^{e}$ s and $\nabla$ denoting the gradient operator $(\partial / \partial x, \partial / \partial y)^{T}$, the problem can be summarized as:

(1) Helmholtz equation: $\nabla^{2} u+k^{2} u=0 \quad$ in all $\Omega^{e}$

(2) Essential boundary condition: $u=\bar{u}$ and $\delta u=0 \quad$ on all $\Gamma_{u}^{e}$

(3) Robin boundary condition: $\bar{\alpha} u+v \mathbf{n}^{T} \nabla u=\bar{t} \quad$ on all $\Gamma_{r}^{e}$

(4) Compatibility condition: $u^{+}=u^{-}$and $\delta u^{+}=\delta u^{-} \quad$ on all $\Gamma_{m}^{e}$

(5) Reciprocity condition: $\left(v \mathbf{n}^{T} \nabla u\right)^{+}+\left(v \mathbf{n}^{T} \nabla u\right)^{-}=0 \quad$ on all $\Gamma_{m}^{e}$

in which $\nabla^{2}=\nabla^{T} \nabla$ is the Laplace operator, $\mathbf{n}$ is the outward unit normal vector to the element boundary, $k$ is the wavenumber, $\delta$ is the variational symbol and $\Gamma_{m}^{e}$ is the inter-element boundary. Quantities with overbars are prescribed in the boundary conditions. Moreover, ( $)^{+}$and ( ) denote the braced quantities at the two sides of $\Gamma_{m}^{e}$. When homogeneous medium problems are considered, $v$ can simply be taken to be unity. However, when $u$ is the spatial amplitude of the pressure in steady-state acoustics, it should be taken to be the specific volume, so that (5) would be the continuity condition of the particle velocity component normal to the inter-element boundary of the adjacent elements which may model different media.

It will be assumed as usual that element boundary $\partial \Omega^{e}$ can be partitioned into the nonoverlapping portions $\Gamma_{u}^{e}, \Gamma_{r}^{e}$ and $\Gamma_{m}^{e}$, i.e.

$$
\Gamma_{u}^{e} \cup \Gamma_{r}^{e} \cup \Gamma_{m}^{e}=\partial \Omega^{e} \quad \text { and } \quad \Gamma_{u}^{e} \cap \Gamma_{r}^{e}=\Gamma_{r}^{e} \cap \Gamma_{m}^{e}=\Gamma_{m}^{e} \cap \Gamma_{u}^{e}=\text { null. }
$$

Note worthily, Robin boundary condition which is also known the impedance boundary condition degenerates into the natural boundary condition when $\bar{\alpha}$ vanishes.

The elemental functional for the conventional finite element formulation of the Helmholtz problem is:

$$
\Pi^{e}=\frac{1}{2} \int_{\Omega^{e}} v\left((\nabla u)^{T} \nabla u-k^{2} u^{2}\right) d \Omega+\int_{\Gamma_{r}^{e}}\left(\frac{1}{2} \bar{\alpha} u^{2}-\bar{t} u\right) d \Gamma
$$

in which $u$ is obtained from the conventional nodal interpolation and $v$ is assumed to be constant within each of the elements. Conditions (2) and (4) can readily be satisfied by the nodal interpolated $u$ and are taken as prerequisites. The global functional for the problem domain is equal to the sum of all $\Pi^{e} s$. By invoking (2), Eq.(2) and the divergence theorem, variation of Eq.(3) can be written as:

$$
\delta \Pi^{e}=-\int_{\Omega^{e}} v\left(\nabla^{2} u+k^{2} u\right) \delta u d \Omega+\int_{\Gamma_{r}^{e}}\left(v \mathbf{n}^{T} \nabla u+\bar{\alpha} u-\bar{t}\right) \delta u d \Gamma+\int_{\Gamma_{m}^{e}}\left(v \mathbf{n}^{T} \nabla u\right) \delta u d \Gamma
$$

The first and second integrals enforce (1) and (3), respectively. With (4) satisfied, the last integral when considered jointly with those of the neighborhood elements enforces (5). 
Figure 1 shows a general three-node element. Using the area coordinates $(s, t) \in[0,1]$, the interpolated $u$ is:

$$
u=[1-s-t, s, t]\left\{\begin{array}{l}
u_{1} \\
u_{2} \\
u_{3}
\end{array}\right\}=\mathbf{N q}
$$
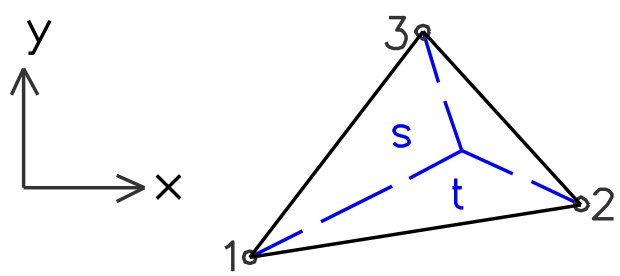

Figure 1. The three-node triangular element: $s$ and $t \in[0,1]$ are the area coordinates.

In the equation, $u_{i}$ 's are the nodal unknowns, $\mathbf{N}$ is the interpolation matrix and $\mathbf{q}$ is the vector of element nodal unknowns. With Eq.(5) substituted into Eq.(3),

$$
\Pi^{e}=\frac{1}{2} \mathbf{q}^{T}\left(\mathbf{K}_{c}+\mathbf{R}\right) \mathbf{q}-\mathbf{f}^{T} \mathbf{q}
$$

in which

$$
\mathbf{K}_{c}=\int_{\Omega^{e}} v\left((\nabla \mathbf{N})^{T}(\nabla \mathbf{N})-k^{2} \mathbf{N}^{T} \mathbf{N}\right) d \Omega, \mathbf{R}=\int_{\Gamma_{r}^{e}} \bar{\alpha} \mathbf{N}^{T} \mathbf{N} d \Gamma \quad \text { and } \quad \mathbf{f}=\int_{\Gamma_{t}^{e}} \mathbf{N}^{T} \bar{t} d \Gamma .
$$

Among them, $\mathbf{K}_{c}$ is the element matrix.

\section{Multi-Field Finite Element Formulation}

The following elemental multi-field functional [9-11] can be formed by employing two Helmholtz variables $u_{b}$ and $u_{d}$ :

$$
\Pi_{h}^{e}=\frac{1}{2} \int_{\Omega^{e}} v\left(\left(\nabla u_{d}\right)^{T} \nabla u_{d}-k^{2} u_{d}^{2}\right) d \Omega+\int_{\partial \Omega^{e}}\left(v \mathbf{n}^{T} \nabla u_{d}\right)\left(u_{b}-u_{d}\right) d \Gamma+\int_{\Gamma_{r}^{e}}\left(\frac{1}{2} \bar{\alpha} u_{b}^{2}-\bar{t} u_{b}\right) d \Gamma .
$$

In the functional, $u_{b}$, which needs to be defined along the boundary only, and $u_{d}$ are the boundary and domain variables, respectively. Obviously, Eq.(7) degenerates into Eq.(3) when $u_{b}$ equals $u_{d}$. Again, the global functional equals to the sum of all $\Pi_{h}^{e}$ s. Variation of $\Pi_{h}^{e}$ is:

$$
\begin{gathered}
\delta \Pi_{h}^{e}=\int_{\Omega^{e}} v\left(\left(\nabla u_{d}\right)^{T} \nabla \delta u_{d}-k^{2} u_{d} \delta u_{d}\right) d \Omega+\int_{\partial \Omega^{e}}\left(\left(v \mathbf{n}^{T} \nabla \delta u_{d}\right)\left(u_{b}-u_{d}\right)+\left(v \mathbf{n}^{T} \nabla u_{d}\right)\left(\delta u_{b}-\delta u_{d}\right)\right) d \Gamma \\
\quad+\int_{\Gamma_{r}^{e}}\left(\bar{\alpha} u_{b}-\bar{t}\right) \delta u_{b} d \Gamma
\end{gathered}
$$

By invoking the divergence theorem and assuming that $u_{d}$ satisfies (2) and (4) as prerequisites, 


$$
\begin{array}{rl}
\delta \Pi_{h}^{e}=-\int_{\Omega^{e}} & v\left(\nabla^{2} u_{d}+k^{2} u_{d}\right) \delta u_{d} d \Omega+\int_{\partial \Omega^{e}}\left(v \mathbf{n}^{T} \nabla \delta u_{d}\right)\left(u_{b}-u_{d}\right) d \Gamma+\int_{\Gamma_{r}^{e}}\left(v \mathbf{n}^{T} \nabla u_{d}+\bar{\alpha} u_{b}-\bar{t}\right) \delta u_{b} d \Gamma \\
& \quad+\int_{\Gamma_{m}^{e}}\left(v \mathbf{n}^{T} \nabla u_{d}\right) \delta u_{b} d \Gamma
\end{array}
$$

It can be seen that the second integral enforces the equality of $u_{b}$ and $u_{d}$ along the element boundary $\partial \Omega^{e}$. The first, third and fourth integrals enforce respectively (1), (3) and (5) with $u$ replaced by $u_{d}$. If $u_{d}$ satisfies the Helmholtz equation, i.e. $\nabla^{2} u_{d}+k^{2} u_{d}=0$, Eq.(7) can be expressed as:

$$
\Pi_{h}^{e}=\int_{\partial \Omega^{e}}\left(-\frac{1}{2}\left(v \mathbf{n}^{T} \nabla u_{d}\right) u_{d}+\left(v \mathbf{n}^{T} \nabla u_{d}\right) u_{b}\right) d \Gamma+\int_{\Gamma_{r}^{e}}\left(\frac{1}{2} \bar{\alpha} u_{b}^{2}-\bar{t} u_{b}\right) d \Gamma
$$

which only involves boundary integrals. The interpolation for $u$ in Eq.(5) is equally applicable to $u_{b}$, i.e.

$$
u_{b}=\mathbf{N q}
$$

Now, let the domain variable be expressed as:

$$
u_{d}=\mathbf{P} \boldsymbol{\beta}
$$

where $\mathbf{P}$ is the shape function matrix and $\boldsymbol{\beta}$ is the vector of coefficients. With Eq.(11) and Eq.(12) substituted into Eq.(10),

$$
\Pi_{h}^{e}=-\frac{1}{2} \boldsymbol{\beta}^{T} \mathbf{H} \boldsymbol{\beta}+\boldsymbol{\beta}^{T} \mathbf{G} \mathbf{q}+\frac{\mathbf{1}}{\mathbf{2}} \mathbf{q}^{\mathrm{T}} \mathbf{R} \mathbf{q}-\mathbf{f}^{T} \mathbf{q}
$$

in which

$$
\mathbf{H}=\int_{\partial \Omega^{e}}\left(v \mathbf{n}^{T} \nabla \mathbf{P}\right)^{T} \mathbf{P} d \Gamma \text { and } \mathbf{G}=\int_{\partial \Omega^{e}}\left(v \mathbf{n}^{T} \nabla \mathbf{P}\right)^{T} \mathbf{N} d \Gamma .
$$

Furthermore, the H-matrix should be symmetric but it may lose its symmetry under numerical integration. In our implementation, $\mathbf{H}$ is symmetrized by averaging itself and its transpose. The stationary condition of Eq.(13) with respect to $\beta$ yields:

$$
\mathbf{H} \boldsymbol{\beta}=\mathbf{G q} \quad \text { or } \quad \boldsymbol{\beta}=\mathbf{H}^{-1} \mathbf{G q}
$$

with which $\boldsymbol{\beta}$ can be condensed from $\Pi_{h}^{e}$ and the latter becomes:

$$
\Pi_{h}^{e}=\frac{1}{2} \mathbf{q}^{T}\left(\mathbf{G}^{T} \mathbf{H}^{-1} \mathbf{G}+\mathbf{R}\right) \mathbf{q}-\mathbf{f}^{T} \mathbf{q}=\frac{1}{2} \mathbf{q}^{T}\left(\mathbf{K}_{h}+\mathbf{R}\right) \mathbf{q}-\mathbf{f}^{T} \mathbf{q}
$$

where $\mathbf{K}_{h}$ is self-defined and is the element matrix of the multi-field finite element model. Same as the conventional element matrix $\mathbf{K}_{c}, \mathbf{K}_{h}$ can also be incorporated into the standard finite element program framework.

In designing multi-field elements for stress/structural analyses, the two common guidelines on the selection of the stress (domain) modes are that the element should be rank sufficient and invariant $[21,22,25,26]$. Furthermore, the optimal number of stress modes in the hybrid-stress element is often considered to be the minimal number that can secure the rank sufficiency. This 
point is echoed in the recent attempt in deriving quadrilateral elements [10]. While this section outlines the principle to formulate the multi-field element model for the plane Helmholtz problem, this and the subsequent sections will present three-node triangular element models which make use of the Bessel and plane-wave solutions of the Helmholtz equation.

\section{Domain Modes from Bessel-Solution Set}

This section considers the domain modes selected from the Bessel-solution set. The element to be proposed is not intended to equip with ad hoc features to tackle the singularity of the Helmholtz variable or its derivatives within the element domain or on the element boundary. After shifting the coordinate origin for defining the domain modes to the element centroid

$$
\left(x_{c}, y_{c}\right)=\left(\frac{x_{1}+x_{2}+x_{3}}{3}, \frac{y_{1}+y_{2}+y_{3}}{3}\right)
$$

for securing the translational invariance $[10,21,22]$, the solution set can be restricted to the nonsingular Bessel functions of the first kind, i.e.

$$
\left\{J_{m}(k \widehat{r}) \exp ( \pm i m \hat{\theta}), m=0,1,2, \ldots\right\}
$$

where

$$
\hat{x}=x-x_{c}, \quad \hat{y}=y-y_{c}, \quad \widehat{r}^{2}=\widehat{x}^{2}+\widehat{y}^{2}, \quad \hat{\theta}=\arctan (\hat{y}, \widehat{x}) .
$$

and those of the second kind need not be considered. For the present element, the first three modes are employed. In other word, the shape function matrix for the domain modes is:

$$
\mathbf{P}=\left[\begin{array}{lll}
J_{0}(k \widehat{r}) \quad J_{1}(k \widehat{r}) \exp (i \widehat{\theta}) \quad J_{1}(k \widehat{r}) \exp (-i \widehat{\theta})
\end{array}\right]
$$

or, without changing the basis,

$$
\mathbf{P}=\left[\begin{array}{lll}
J_{0}(k \widehat{r}) \quad J_{1}(k \widehat{r}) \cos \widehat{\theta} \quad J_{1}(k \widehat{r}) \sin \widehat{\theta}
\end{array}\right]
$$

which is complete to first order Bessel function of the first kind. Hence, rotational invariance $[10,21,22]$ can also be secured. When $k$ and the boundary conditions are real, complex data type and computation can be avoided by the latter format of the P-matrix which is real.

\section{Radial Basis Function as Domain Modes}

Similar to the radial basis function technique [23,24] in which $J_{0}$ 's with different origins are employed, the shape function matrix for the domain modes of the second three-node triangular element is chosen to be:

$$
\mathbf{P}=\left[\begin{array}{lll}
J_{0}\left(k r_{1}\right) & J_{0}\left(k r_{2}\right) & J_{0}\left(k r_{3}\right)
\end{array}\right]
$$

where $r_{i}^{2}=\left(x-x_{i}\right)^{2}+\left(y-y_{i}\right)^{2}$ and $\left(x_{i}, y_{i}\right)$ are the nodal coordinates. There are two reasons for picking the nodes as the origins of $J_{0}$. Firstly, the boundary variable can only assume its extreme values at the nodes. It is consistent to the present choice as extreme values of $\pm J_{0}$ occur at the nodes. Secondly, the contours of $J_{0}\left(k r_{i}\right)$ are perpendicular to the two element edges defining the $i$ th node. In other words, along each of the element edge, two out of the three entries in $\mathbf{n}^{T} \nabla \mathbf{P}$ 
vanishes. This property can be employed to reduce the computational cost of forming the G- and H-matrices defined under Eq.(13) [28]. Note worthily, a mathematical proof on the completeness of the radial basis function technique using $J_{0}$ is pending. However, the elements derived in this and last sections yield practically identical predictions.

It is equally possible to use the fundamental solution, i.e. $Y_{0}$ or the singular zeroth order Bessel function of the second kind, outside the element as the domain modes. However, the computational advantage due to zero $\mathbf{n}^{T} \nabla \mathbf{P}$ would be lost. Moreover, the solution would be sensitive to the distance from the element boundary to the origins of the $Y_{0}$ 's $[23,24]$.

\section{Plane-Wave Modes Vanish along Element Edges}

For completeness, forward and backward plane-wave solutions are employed in pair in most, if not all, advanced finite element techniques. On the other hand, the nodal interpolation does not contain any directional property where as the variational functional in Eq.(7) enforces the equality of the domain field and boundary field which is constructed by nodal interpolation. Hence, it may be advantageous to employ domain modes that are similar to the boundary modes and disregard the pairwise inclusion of the plane-wave solutions. In this light, the following set of plane-wave modes are identified:

$$
\sin \left[k\left(\mathbf{r}-\mathbf{r}_{1}\right)^{T} \boldsymbol{\varphi}_{1}\right], \sin \left[k\left(\mathbf{r}-\mathbf{r}_{2}\right)^{T} \boldsymbol{\varphi}_{2}\right] \text { and } \sin \left[k\left(\mathbf{r}-\mathbf{r}_{3}\right)^{T} \boldsymbol{\varphi}_{3}\right]
$$

where $\mathbf{r}_{i}=\left\{x_{i}, y_{i}\right\}^{T}$ and $\varphi_{i}$ is the unit vector defined at the $i$-th node of the element in Figure 2(a). Taking the first function as an example, it is identically zero along the edge defined by nodes 1 and 2; when the nodal spacing is considerably smaller than the wavelength, the function increases monotonically and very much linearly from zero at nodes 1 and 2 . These geometric properties are similar to those of the interpolation function of node 3 .

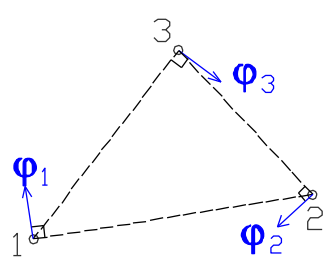

(a)

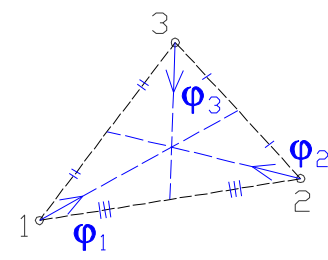

(d)

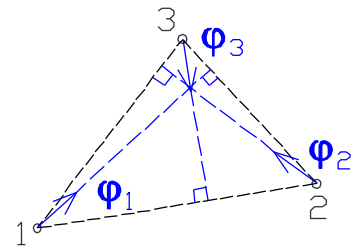

(b)

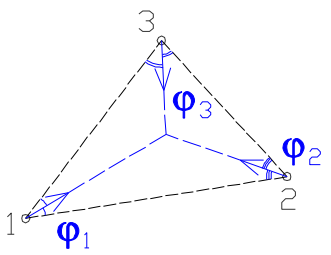

(e)

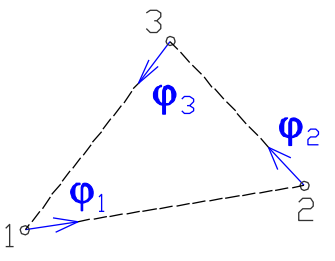

(c)

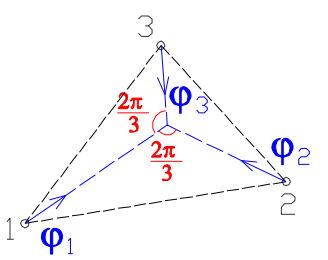

(f)

Figure 2. Directions of plane-wave modes for (a) edge modes, (b) node-orthocenter modes, (c) node-node modes, (d) node- centroid modes, (e) node- incenter modes, and (f) node-Fermat modes. 
As each of the above modes vanishes at an element edge, they would be termed as edge modes for simplicity. With them taken to be domain modes, the resulting multi-field model is more accurate than the ones described in Sections 4 and 5.

\section{Plane-Wave Modes Vanish at Element Nodes}

With the encouraging result of the edge modes, other modes which can be described by using different unit vectors in (21) are attempted. They include the node-orthocenter modes, node-node modes, node-centroid modes, node-incenter modes and node-Fermat modes. The unit vectors are portrayed in Figures 2(b), 2(c), 2(d), 2(e) and 2(f), respectively. They are self-defined by the nomenclature. For instance, the node-orthocenter modes employ the unit vectors which point from the nodes to the orthocenter which is the intersection of the altitudes. On the other hand, centroid and incenter are the intersection of the medians and the intersection of the angular bisectors, respectively; the three lines connecting the Fermat point to the corners of the triangle equally divide the $2 \pi$ angular space [29].

Unlike the edge modes, each of the plane-wave modes considered in this section vanish at only an element node but not an element edge. Hence, they would be termed as nodal modes for simplicity.

\section{Numerical Examples}

In this section, the numerical predictions of the following three-node triangular element models are presented and compared:

Conv - the conventional single-field model, see Section 2;

Bes - the hybrid-Trefftz element formulated with the Bessel solution modes in Eq.(19);

RBF - the multi-field element formulated with the distributed Bessel solution modes in Eq.(20);

Edg - the multi-field element formulated with the edge modes, see Figure 2(a);

Nod - the multi-field element formulated with the node-node modes, see Figure 2(c).

Among the five multi-field models formulated with nodal modes, see Section 7, "Nod" which employs the node-node modes yields consistently more accurate predictions than the others whose results are not included for conciseness and graphical clarity. Moreover, along each of the element edge, one out of the three entries in $\mathbf{n}^{T} \nabla \mathbf{P}$ of "Nod" vanishes. Same as "RBF", this feature can be employed to reduce the computational cost of forming the G- and H-matrices defined under Eq.(13).

Unless stated otherwise, the boundary and domain integrals are evaluated by second order Gaussian quadrature and the three-point domain rule, respectively. In the latter, the sampling points are $(1 / 6,1 / 6),(2 / 3,1 / 6)$ and $(1 / 6,2 / 3)$ with respect to the area coordinates $(s, t)$. For comparing the element predictions, the following "normalized error" and "relative error" with respect to the error of "Conv" are defined: 


$$
\text { Normalized error }=\left(\frac{\int_{\Omega}\left|u-u^{\text {exact }}\right|^{2} d \Omega}{\int\left|u^{\text {exact }}\right|^{2} d \Omega}\right)^{1 / 2}, \text { Relative error }=\left(\frac{\int_{\Omega}\left|u-u^{\text {exact }}\right|^{2} d \Omega}{\int\left|u_{\Omega}^{\text {Conv }}-u^{\text {exact }}\right|^{2} d \Omega}\right)^{1 / 2}
$$

where || returns the magnitude of the embraced complex quantity. The errors will be evaluated by nodal integration with the area factor taken to be one-third of the total area in the elements sharing the node. Furthermore, the number of nodal spacings per wavelength

$$
N_{n}=\frac{\text { wave length }}{\text { nodal spacing }}=\frac{2 \pi}{\operatorname{Re}(k) \cdot h}
$$

may be specified. In the expression, $h$ denotes the nodal spacing. For the conventional linear element model, many literatures recommend $N_{n}>10$ (see, e.g., [11,14] among others).

\subsection{Integration schemes}

The edge lengths of the element in Figure 3 are 1, $\sqrt{ } 0.97$ and $\sqrt{ } 1.17$ which give an average nodal spacing of $\sim 1.01$. Two wavenumbers $k=0.6(1+i)$ and $1.25(1+i)$ are considered. The relevant $N_{n}$ s are approximately equal to 10 and 5, respectively. Let $|\alpha|$ denote the eigenvalue magnitude of the element matrix $\mathbf{K}_{c}$ or $\mathbf{K}_{h},|\alpha|_{\max },|\alpha|_{\min }$ and the condition number $N_{c}$ defined as $\left.|\alpha|_{\max }|| \alpha\right|_{\min }$ are computed. Table 1 lists the computed values of the elements when different integration schemes [27] are employed. When the domain integration scheme for "Conv" changes from the three-point to six-point rule and the boundary integration scheme for the multi-field models change from second to third order quadrature, the changes in $N_{c}$ 's for all elements are less than $0.5 \%$. These justify the employed integration schemes outlined at the beginning of Section 6 . They are sufficiently accuracy even when $N_{n}$ falls to 5 . It can be noted that $N_{c}$ of "Edg" changes considerably when the integration scheme changes from 1st to 2 nd order quadrature.

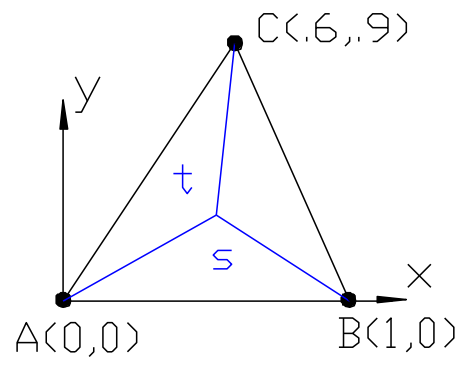

(a)

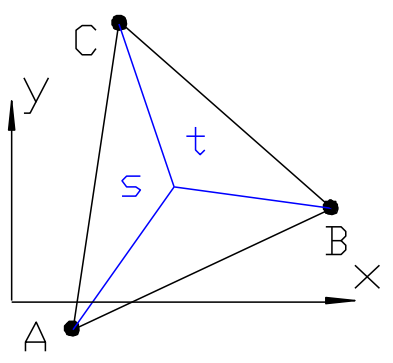

(b)

Figure 3. The single-element problem for examining the condition number and invariance of the element matrices. (b) is obtained from (a) by rigid body translation and rotation. Moreover, $\mathrm{A}$ is the first element node in (a) whereas $\mathrm{C}$ is the first element node in (b). 
Table 1. $|\alpha|_{\max },|\alpha|_{\min }$ and $N_{c}$ of the proposed elements under different orders.

\begin{tabular}{|c|c|c|c|c|c|c|c|c|c|c|}
\hline \multirow{2}{*}{$\begin{array}{l}\operatorname{Re} \\
(k)\end{array}$} & \multirow{2}{*}{ model } & \multicolumn{3}{|c|}{$\begin{array}{l}\text { One-point domain rule } \\
\text { or } 1 \text { st order quadrature }\end{array}$} & \multicolumn{3}{|c|}{$\begin{array}{l}\text { three-point domain rule } \\
\text { or } 2 \text { nd order quadrature }\end{array}$} & \multicolumn{3}{|c|}{$\begin{array}{l}\text { Six-point domain rule } \\
\text { or 3rd order quadrature }\end{array}$} \\
\hline & & $|\alpha|_{\max }$ & $|\alpha|_{\text {min }}$ & $N_{c}$ & $|\alpha|_{\max }$ & $|\alpha|_{\min }$ & $N_{c}$ & $|\alpha|_{\max }$ & $|\alpha|_{\min }$ & $N_{c}$ \\
\hline \multirow{5}{*}{0.6} & Conv & 0.976 & 0.108 & 9.037 & 0.976 & 0.108 & 9.041 & 0.976 & 0.108 & 9.041 \\
\hline & Bes & 0.976 & 0.108 & 9.039 & 0.977 & 0.108 & 9.044 & 0.977 & 0.108 & 9.043 \\
\hline & $\mathrm{RBF}$ & 0.976 & 0.108 & 9.043 & 0.977 & 0.108 & 9.046 & 0.977 & 0.108 & 9.044 \\
\hline & Edg & 0.976 & 0.081 & 12.05 & 0.977 & 0.108 & 9.053 & 0.978 & 0.108 & 9.053 \\
\hline & Nod & 0.975 & 0.108 & 9.024 & 0.980 & 0.108 & 9.073 & 0.980 & 0.108 & 9.073 \\
\hline \multirow{5}{*}{1.25} & Conv & 0.976 & 0.469 & 2.082 & 0.983 & 0.469 & 2.097 & 0.983 & 0.469 & 2.097 \\
\hline & Bes & 0.978 & 0.468 & 2.089 & 0.986 & 0.467 & 2.110 & 0.987 & 0.468 & 2.108 \\
\hline & $\mathrm{RBF}$ & 0.984 & 0.467 & 2.107 & 0.990 & 0.467 & 2.119 & 0.988 & 0.468 & 2.111 \\
\hline & Edg & 0.975 & 0.351 & 2.778 & 1.002 & 0.467 & 2.148 & 1.007 & 0.468 & 2.150 \\
\hline & Nod & 0.957 & 0.468 & 2.044 & 1.033 & 0.467 & 2.211 & 1.036 & 0.468 & 2.212 \\
\hline
\end{tabular}

1: used by "Conv" for domain integration. 2: used by multi-field models for boundary integration.

\subsection{Invariant tests}

The element in Figure 3(b) is obtained from that in Figure 3(a) by translation, rotation and change of connectivity. Other combinations of translation, rotation and connectivity have also been attempted. Under various settings, all the element eigenvalues remain unchanged. The invariance of the elements are confirmed.

\subsection{Plane-wave problems}

Figure 4 depicts a $L \times L$ problem domain modelled by the $4 \times 4$ mesh which contains $2 \times 4 \times 4$ triangular elements and $L$ is set to be 2 . With $v=1$, the entire domain boundary is prescribed with the Robin boundary condition:

$$
\mathbf{n}^{T} \nabla u-i k u=\mathbf{n}^{T} \nabla u^{e x a c t}-i k u^{e x a c t}
$$

in which $\operatorname{Re}(k)=\operatorname{Im}(k)$ and the exact solution is:

$$
u^{\text {exact }}=\exp \left[i k \mathbf{r}^{T} \phi\right]=\exp [i k(x \cos \phi+y \sin \phi)]
$$

where $\phi$ is the wave propagation direction. Owing to the mesh topology and domain geometry, the errrors are symmetric about $\phi=45^{\circ}$ and $135^{\circ}$. Hence, only the errors for $45^{\circ} \leq \phi \leq 135^{\circ}$ are presented. Figures 5 and 6 show the normalized errors for $\operatorname{Re}(k L)=4$ and 16 , respectively. $N_{n}$ is fixed at $3 \pi$ by employing the $6 \times 6$ and $24 \times 24$ meshes, respectively. "Bes", "RBF" and "Edg" produce graphically indistinguishable results which are not separately presented in the figures for clarity. One can see that the the results in the two figures are fairy similar. The same observation 


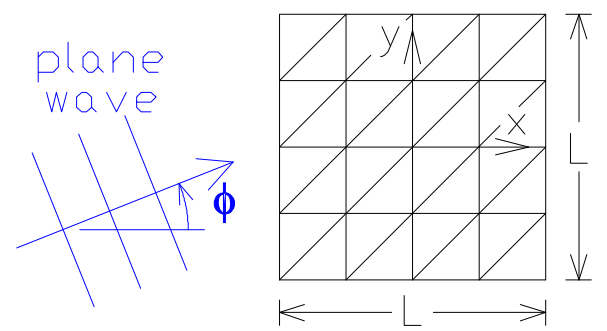

Figure 4 . The plane-wave problem modelled by the uniform $4 \times 4$ mesh.

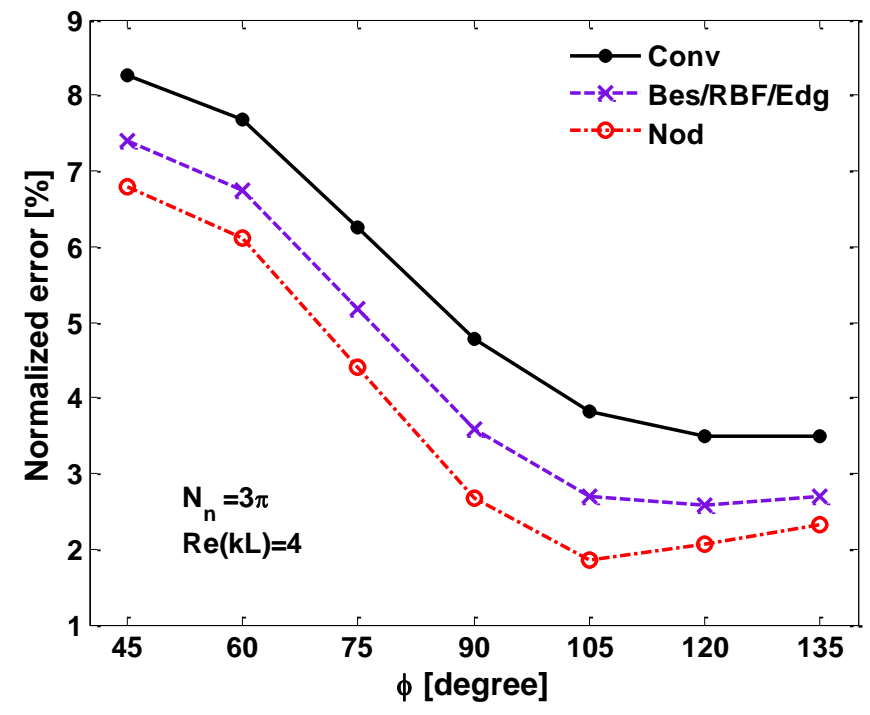

(a)

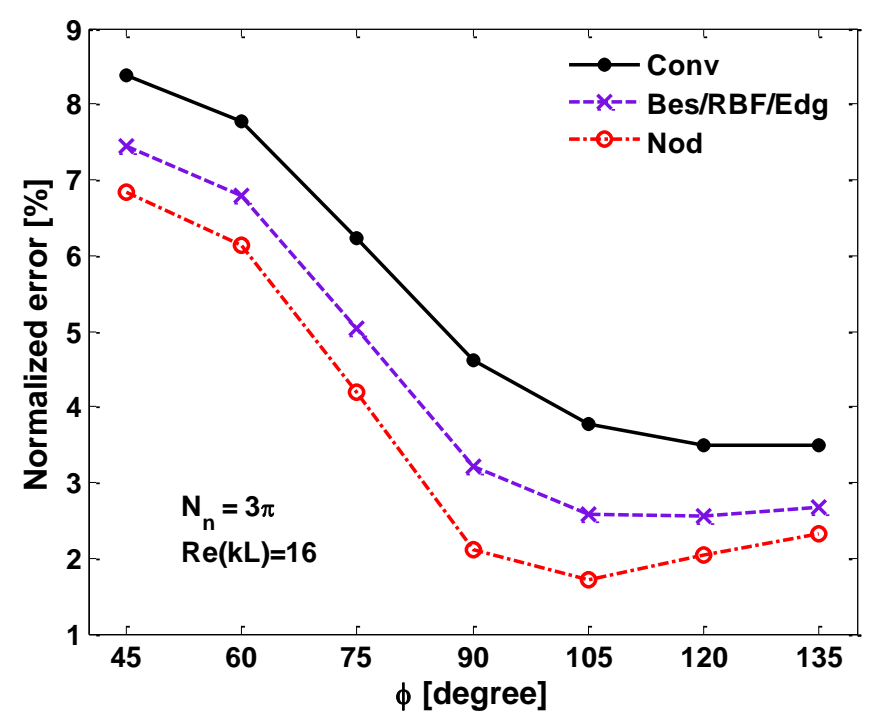

(b)

Figure 5. Normalized errors in the plane-wave problem for (a) $\operatorname{Re}(k) L=4$ and (b) $\operatorname{Re}(k) L=16$. The number of nodal spacings per wavelength $N_{n}$ is fixed at $3 \pi$. 
also applies to the results for $\operatorname{Re}(k L)=8$ and 12 . The average relative errors bearing the following definition:

$$
\text { Average relative error }=\frac{1}{n_{p}} \sum_{i=1}^{n_{p}} \frac{\text { error of the element at the i-th } \phi}{\text { error of "Conv" at the i-th } \phi}
$$

are computed for both the real and imginary parts of the predictions, see Table 2 . In the equation, $n_{p}$ denotes the number of data points. As seen in the table, the average relative errors for "Bes" and "Nod" vary from 0.74 to 0.79 and from 0.58 to 0.66 , respectively. The closeness of the predictions from "Bes", "RBF" and "Edg* can also be noted.

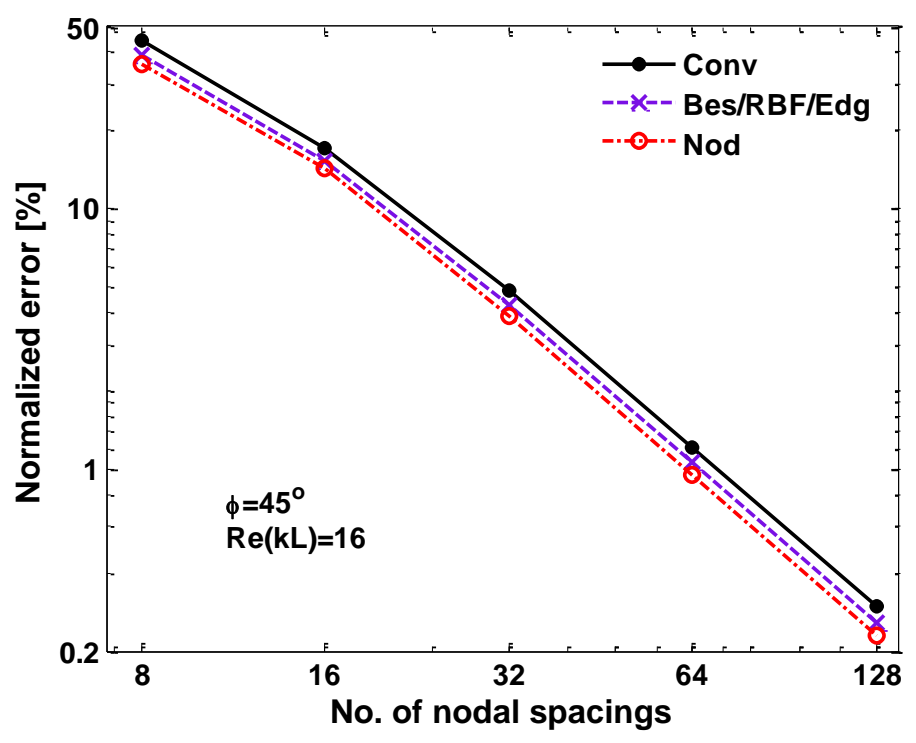

(a)

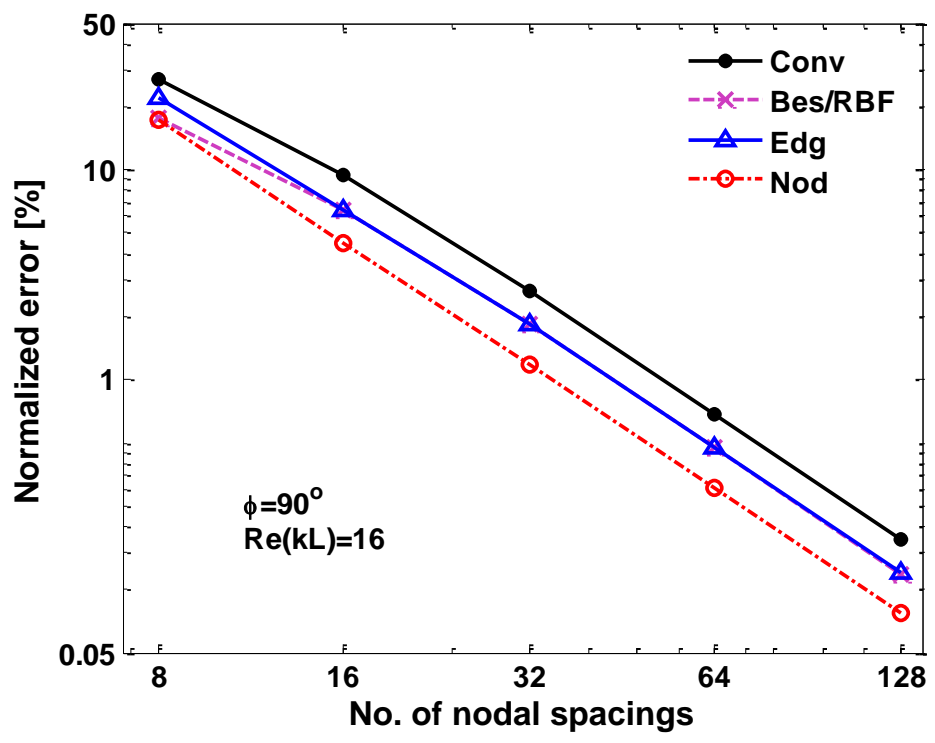

(b) 


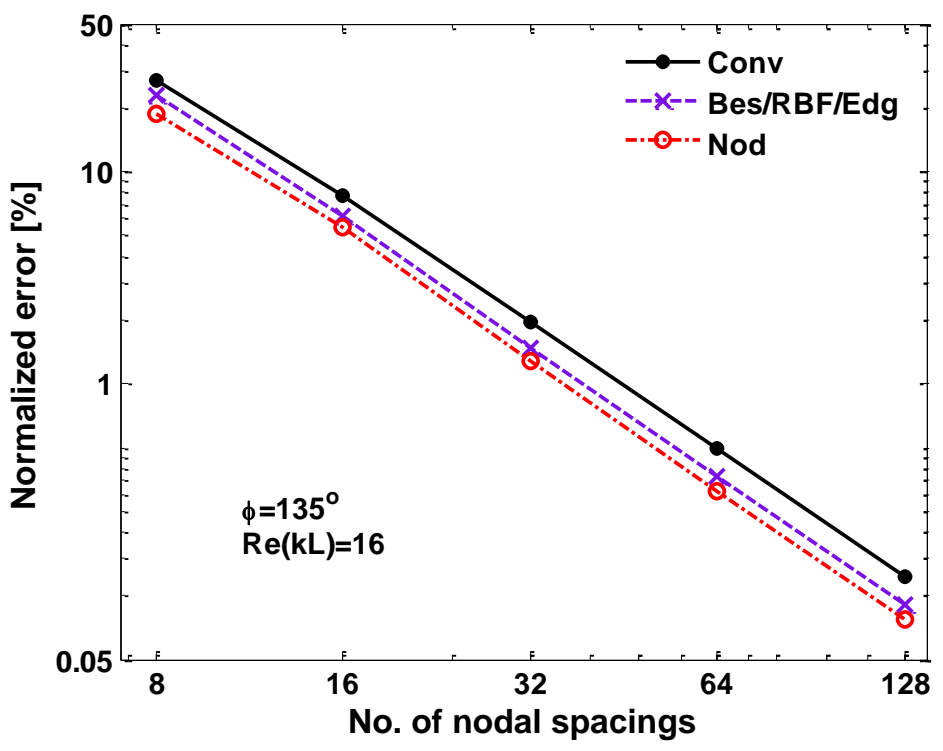

(c)

Figure 6. Convergence studies for the plane-wave problem. The number of elements along a domain edge varies from $8\left(N_{n}=4 \pi\right)$ to $100\left(N_{n}=12.5 \pi\right)$. The wave propagation directions $\phi$ are (a) $45^{\circ}$, (b) $90^{\circ}$ and (c) $135^{\circ}$.

Table 2. Average relative errors for the errors presented in the format of Figure $5\left(n_{p}=7\right)$.

\begin{tabular}{|l|l|l|l|l|l|l|l|l|l|l|l|}
\hline \multirow{2}{*}{$\operatorname{Re}(k L)$} & \multirow{4}{*}{ Mesh } & \multicolumn{4}{|l|}{ Real part of the prediction } & \multicolumn{4}{|l|}{ Imaginary part of the prediction } \\
\cline { 3 - 12 } & Conv & Bes & RBF & Edg & Nod & Conv & Bes & RBF & Edg & Nod \\
\hline 4 & $6 \times 6$ & 1.000 & 0.795 & 0.794 & 0.799 & 0.663 & 1.000 & 0.793 & 0.784 & 0.789 & 0.652 \\
\hline 8 & $12 \times 12$ & 1.000 & 0.787 & 0.784 & 0.791 & 0.651 & 1.000 & 0.755 & 0.748 & 0.753 & 0.597 \\
\hline 12 & $18 \times 18$ & 1.000 & 0.785 & 0.781 & 0.787 & 0.643 & 1.000 & 0.745 & 0.742 & 0.748 & 0.586 \\
\hline 16 & $24 \times 24$ & 1.000 & 0.779 & 0.774 & 0.781 & 0.632 & 1.000 & 0.760 & 0.759 & 0.762 & 0.607 \\
\hline
\end{tabular}

By fixing $\operatorname{Re}(k L)$ to 16 , the number of nodal spacings per domain edge is varied from $8\left(N_{n}=\right.$ $\pi)$ to $128\left(N_{n}=16 \pi\right)$ for convergence studies. Figures 6(a), (b) and (c) show the normalized errors of the elements for $\varphi=45^{\circ}, 90^{\circ}$ and $135^{\circ}$, respectively. The convergence rates of all elements at the three and all other $\varphi$ 's are essentially the same. The behaviour is well-expected as the rate should be controlled by the nodal interpolation order. Table 3 lists the average relative errors for both the real and imginary parts of the predictions at five $\varphi$ 's ranging from $45^{\circ}$ to $135^{\circ}$. As seen in the table, the average relative errors for "Bes" and "Nod" vary from 0.67 to 0.88 and from 0.47 to 0.80 , respectively. 
Table 3. Average relative errors for the errors presented in the format of Figure $6\left(n_{p}=5\right)$.

\begin{tabular}{|c|c|c|c|c|c|c|c|c|c|c|}
\hline \multirow{2}{*}{$\varphi$} & \multicolumn{4}{|c|}{ Real part of the prediction } & \multicolumn{5}{c|}{ Imaginary part of the prediction } \\
\cline { 2 - 12 } & Conv & Bes & RBF & Edg & Nod & Conv & Bes & RBF & Edg & Nod \\
\hline $45^{\circ}$ & 1.000 & 0.889 & 0.894 & 0.879 & 0.803 & 1.000 & 0.864 & 0.856 & 0.884 & 0.795 \\
\hline $67.5^{\circ}$ & 1.000 & 0.796 & 0.795 & 0.823 & 0.703 & 1.000 & 0.854 & 0.854 & 0.859 & 0.755 \\
\hline $90^{\circ}$ & 1.000 & 0.672 & 0.662 & 0.724 & 0.474 & 1.000 & 0.697 & 0.693 & 0.700 & 0.521 \\
\hline $112.5^{\circ}$ & 1.000 & 0.678 & 0.617 & 0.691 & 0.525 & 1.000 & 0.721 & 0.720 & 0.741 & 0.553 \\
\hline $135^{\circ}$ & 1.000 & 0.797 & 0.764 & 0.801 & 0.653 & 1.000 & 0.691 & 0.759 & 0.699 & 0.679 \\
\hline
\end{tabular}

\subsection{Cylindrical-wave problems with one, two and four layers of media}

Figures 7(a), 7(b) and 7(c) show a single-, two- and four-layered cylinders formed by two media "A" and "B" which are non-hatched and hatched, respectively. The outer diameters of the cylinders are 8 and the interfaces between different media, if any, are at radial distances $r=1,2$ and 3 from the centers. The specific volumes and wavenumbers of the media are $v_{\mathrm{A}}=4, v_{\mathrm{B}}=1, k_{\mathrm{A}}$ $=1$ and $k_{\mathrm{B}}=2$. In the innermost or the first layer, the exact solution is:

$$
u_{1}=J_{0}\left(k_{1} r\right)
$$

and the exact solution for the $i$-th layer $(i \geq 2)$ assumes the following form due to axial symmetry:

$$
u_{i}=a_{i} J_{0}\left(k_{i} r\right)+b_{i} Y_{0}\left(k_{i} r\right)
$$

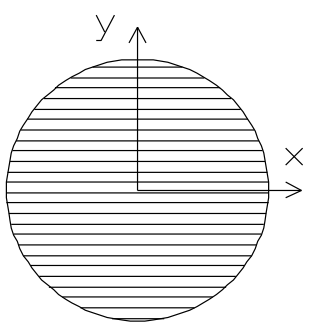

(a)

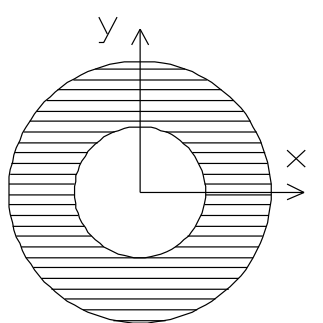

(b)

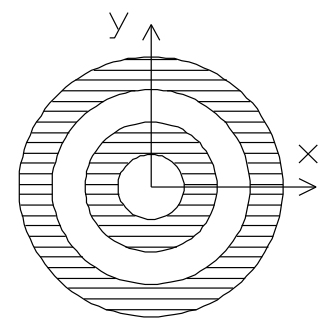

(c)

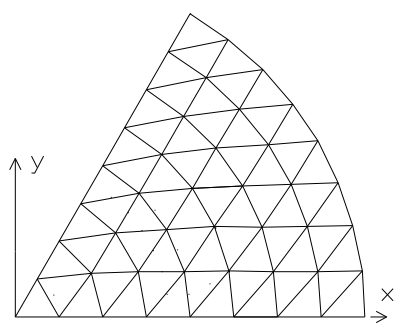

(d)

Figure 7. The $\phi 8$ cylinder with (a) single, (b) two and (c) four layers of media. (d) The mesh with 8 nodal spacings along the $x$-axis for a $\pi / 3$ sectorial subdomain of the $\phi 8$ cylinder.

In the last expression, $Y_{0}$ is the zeroth order Bessel function of the second kind. The coefficients $a_{i}$ and $b_{i}$ can be solved by the compatibility and reciprocity conditions at the interface between the $(i-1)$-th and $i$-th layers, i.e.

$$
u_{i}=u_{i-1}, v_{i} \frac{d u_{i}}{d r}=v_{i-1} \frac{d u_{i-1}}{d r} .
$$

Owing to symmetry, only a $\pi / 3$ sector of the problem domain is considered and Figure $7(\mathrm{~d})$ shows the mesh in which the number of nodal spacings along the $x$-axis equal to $8(h=1 / 2)$. 
Along the circular boundary of the sector $(r=4)$, essential boundary condition is prescribed. The two straight edges of the sector are prescribed with $\mathbf{n}^{T} \nabla u=0$. Three other meshes with $16(h=$ $1 / 4), 24(h=1 / 6)$ and $32(h=1 / 8)$ nodal spacings along the $\mathrm{x}$-axis are also employed.

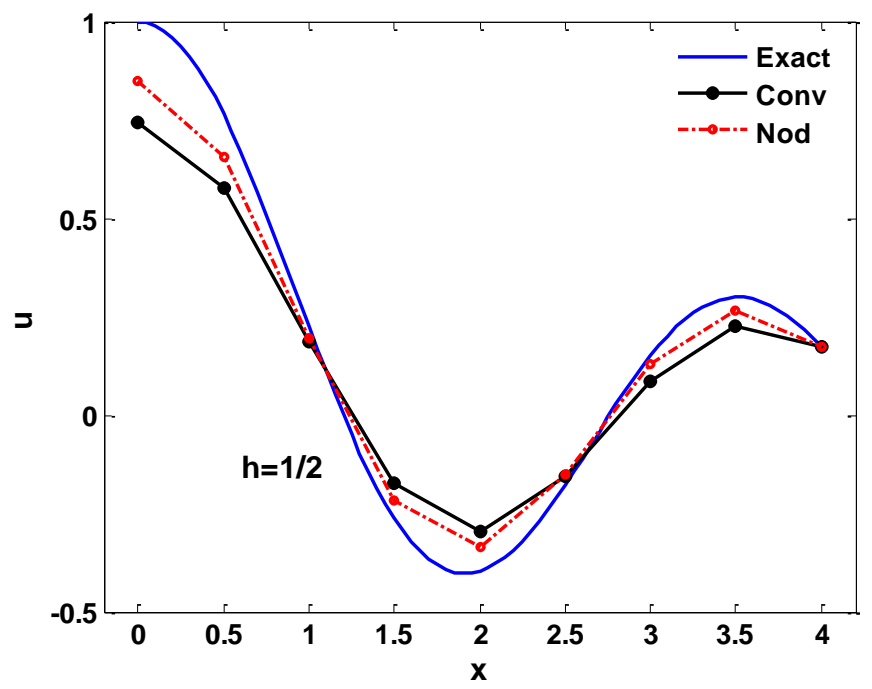

(a)

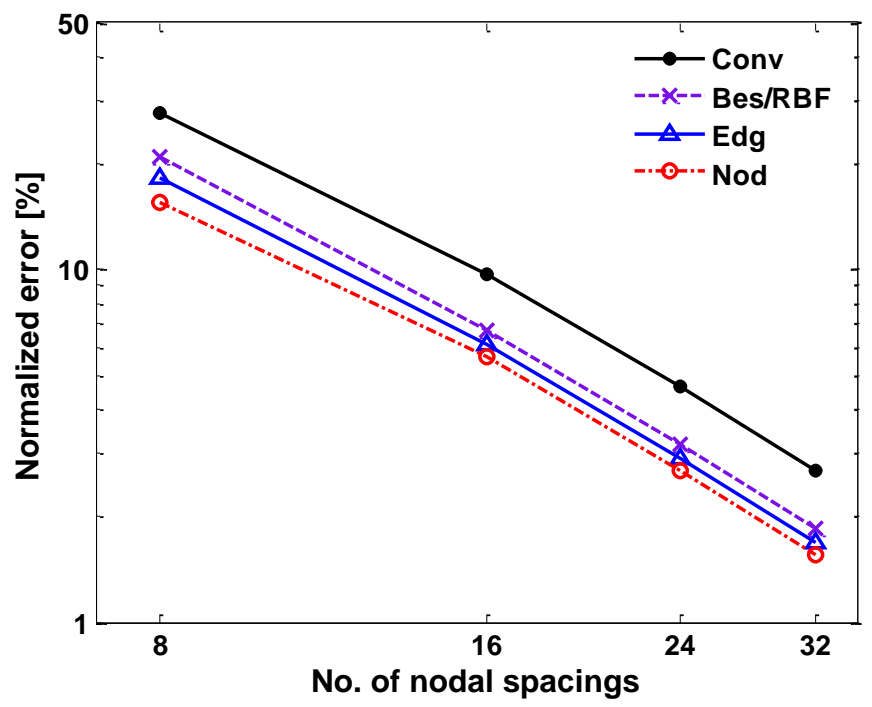

(b)

Figure 8. Nodal predictions along the $\mathrm{x}$-axis and convergence study for the $\phi 8$ cylinder with single layer of media, see Figures 7(a) and 7(d).

Figures 8(a), 9(a) and 10(a) plot the nodal predictions of "Conv" and "Nod" for $h=1 / 2$ as well as the exact solutions along the $x$-axis for the single-, two- and four-layered problems, respectively. For clarity, the predictions of "RBF" and "Edg" are not included in the figures. 
Their predictions, however, are between those of "Conv" and "Nod" as shown in the convergence plots in Figures 8(b), 9(b) and 10(b) for the single-, two- and four-layered problems, respectively. For the four-layered problem, all finite element models produce grahically indistinguishable results in Figure 10(a). Their difference, however, can be seen in Figure 10(b). Table 4 lists the average relative errors of the convergence plots. Among the multi-field models, "Edg" and "Nod" offer the best accuracy.

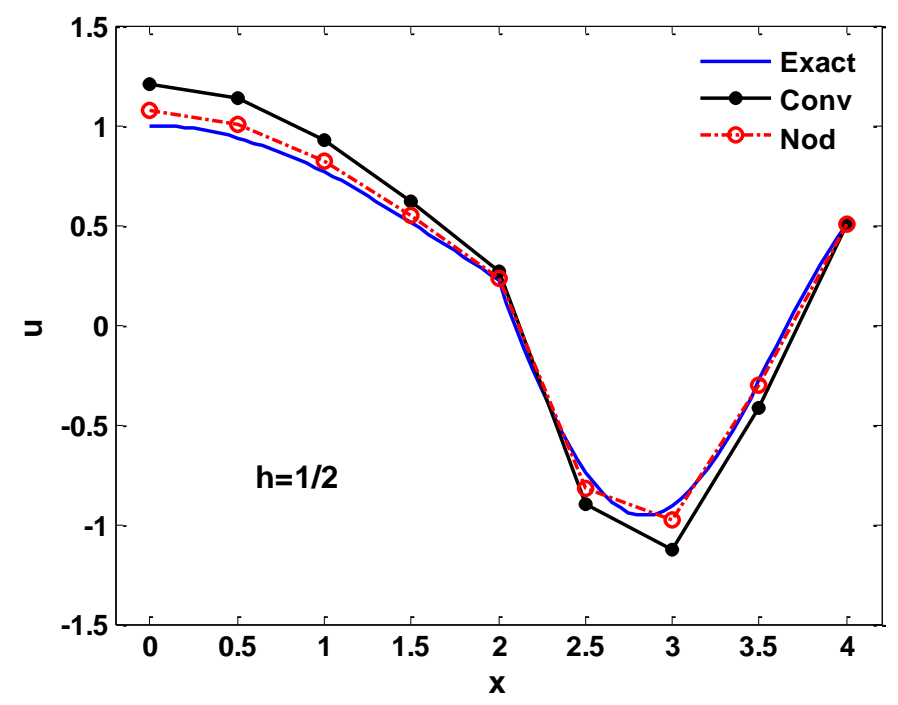

(a)

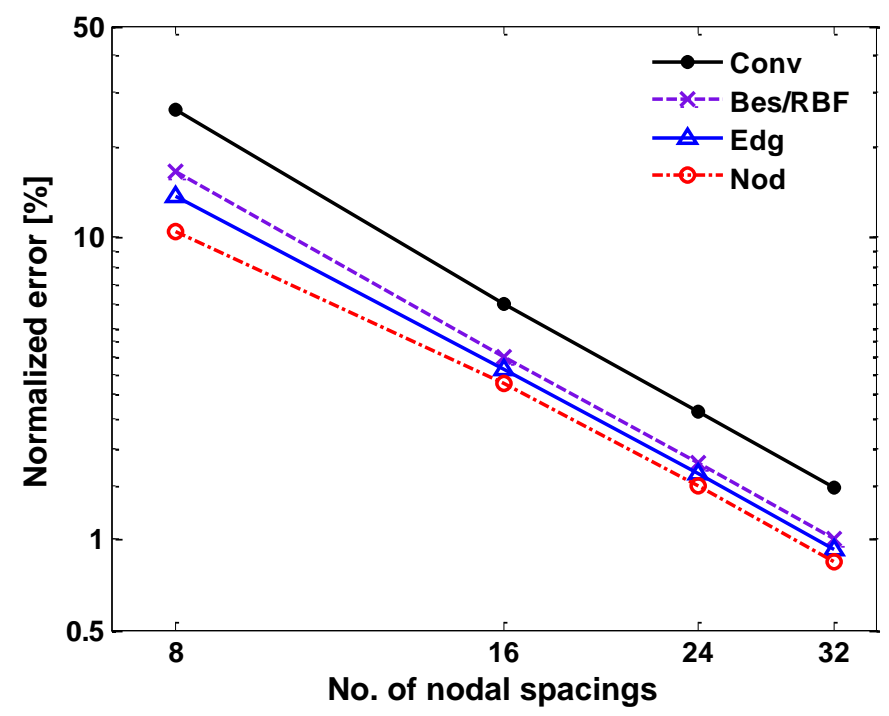

(b)

Figure 9. Nodal predictions along the $\mathrm{x}$-axis and convergence study for the $\phi 8$ cylinder with two layer of media, see Figures 7(b) and 7(d). 


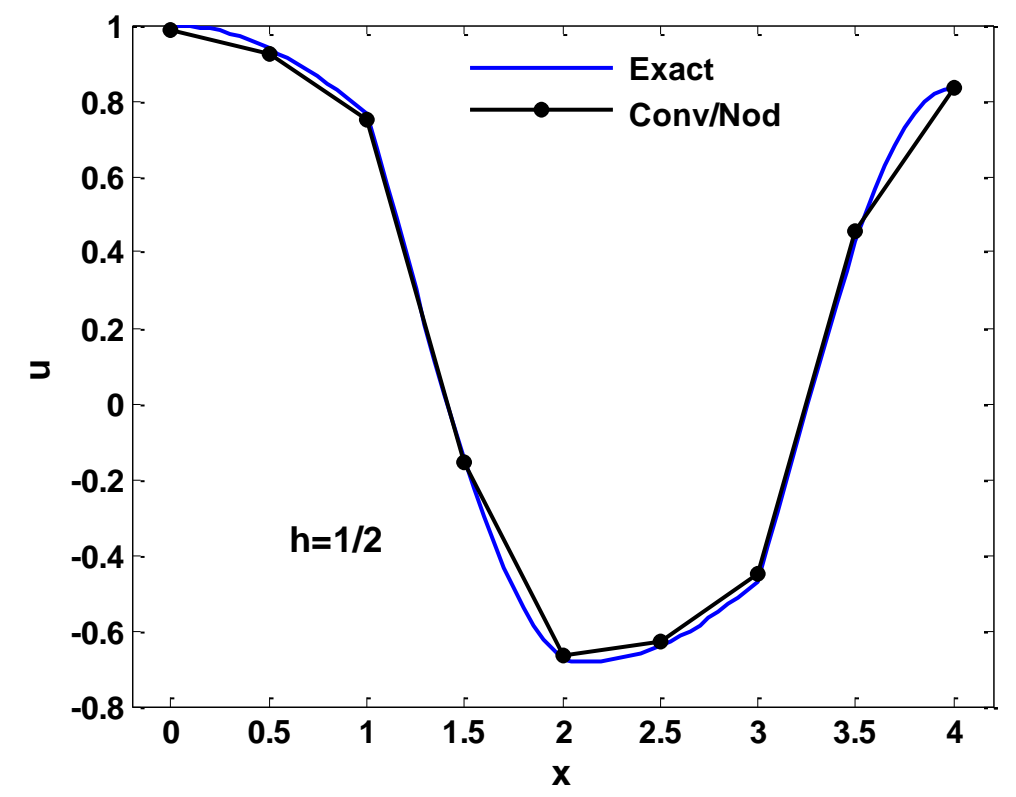

(a)

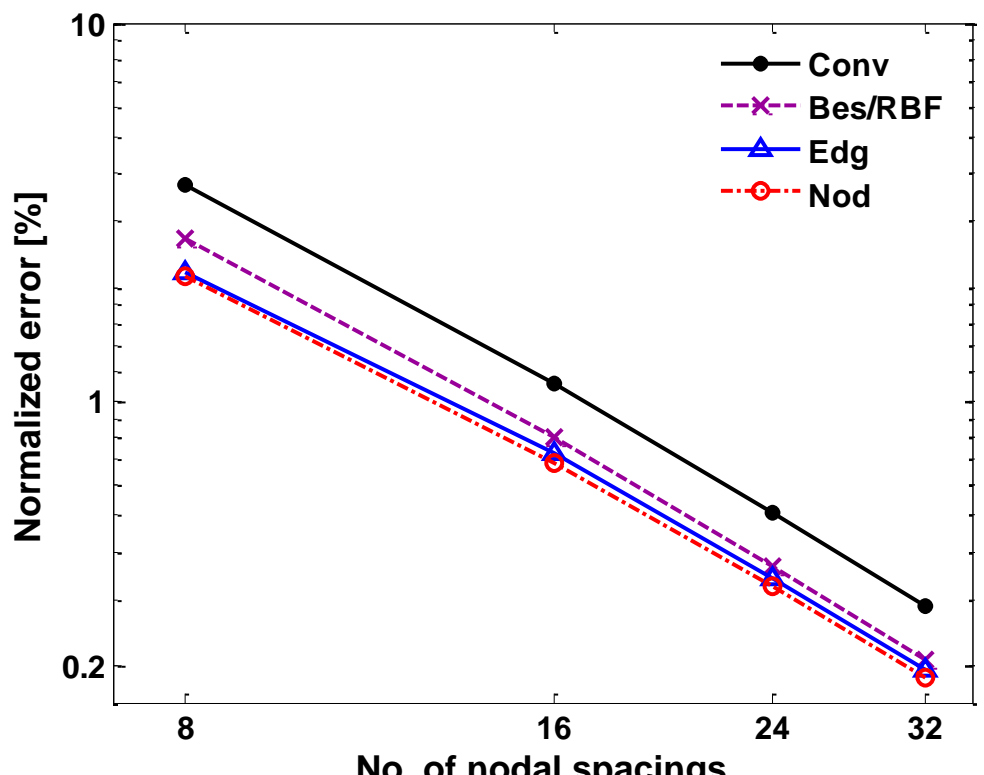

(b)

Figure 10. Nodal predictions along the $\mathrm{x}$-axis and convergence study for the $\phi 8$ cylinder with four layers of media, see Figure 7(c) and 7(d). 
Table 4. Average relative errors for results presented in Figures 8(b), 9(b) and 10(b) $\left(n_{p}=4\right)$.

\begin{tabular}{|c|c|c|c|c|c|}
\hline Number of layers & Conv & Bes & RBF & Edg & Nod \\
\hline 1 & 1 & 0.7044 & 0.7023 & 0.6374 & 0.5757 \\
\hline 2 & 1 & 0.6638 & 0.6608 & 0.5944 & 0.5197 \\
\hline 4 & 1 & 0.7217 & 0.7159 & 0.6448 & 0.6202 \\
\hline
\end{tabular}

\section{Closure}

In this paper, four rank sufficient and invariant three-node triangular finite element models which can readily be incorporated into the standard finite element program framework are devised via a multi-field variational functional. The first element is a typical hybrid-Trefftz element in the sense that the domain modes are truncated from the Bessel solution set. In the second model, the three domain modes are the three zeroth order Bessel functions of the first kind with their origins at the element nodes. For the last two models, the common practice of using both the forward and backward plane-wave solutions is abandoned and the solutions in the form of $\sin \left[k\left(\mathbf{r}-\mathbf{r}_{i}\right)^{T} \boldsymbol{\varphi}_{i}\right]$ in which $\mathbf{r}_{i}$ is the position vector of the $i$-th element node and $\varphi_{i}$ is the traversing direction are adopted. In the third and fourth models, $\varphi_{i}$ is perpendicular to and along the element edge, respectively. Numerical studies indicate that the first two multi-field models always yield graphically indistinguishable results. The fourth model is markedly more accurate than the first three models and, in most examples, is $1 / 3$ to $1 / 2$ more accurate than the conventional single-field model.

Domain modes in the form of $\cos \left[k\left(\mathbf{r}-\mathbf{r}_{i}\right)^{T} \boldsymbol{\varphi}_{i}\right]$ are also attempted. However, the resulting models are even less accurate than the conventional single-field model and sometimes nonconvergent. The observable may be explained by the leading terms of the cos and sin functions which are the constant and linear terms, respectively. The latter fits the linear nodal interpolation functions whereas the employed functional aims to minimize the domain nodal interpolated fields.

\section{Acknowledgment}

This work is supported by the Hong Kong Research Grant Council in the form of a GRF grant (HKU 7161 08E).

\section{References}

1. Zielinski AP, Herrera I. Trefftz method: fitting boundary conditions. Int.J.Numer.Meth.Engng. 24 (1987) 871-891.

2. Cheung YK, Jin WG, Zienkiewicz OC. Solution of Helmholtz equation by Trefftz method. Int.J.Numer. Meth.Engng. 32 (1991) 63-78.

3. Herrera I. Trefftz method: A general theory. Numerical Meth.for Partial Differential Equations 16 (2000) 561-580.

4. Sladek J, Sladek V, Keer RV. Global and local Trefftz boundary integral formulations for sound vibration. Advances in Engineering Software 33 (2002) 469-476. 
5. Chang JR, Liu RF. An asymmetric indirect Trefftz method for solving free-vibration problems. J. Sound \& Vibration 275 (2004) 991-1008.

6. Stojek M. Least square Trefftz-type elements for the Helmholtz equation. Int.J.Numer.Meth. Engng. 41 (1998) 831-849.

7. Stojek M, Markiewicz M, Mahrenholtz O. Diffraction loads on multiple vertical cylinders with rectangular cross section by Trefftz-type finite elements, Computers \& Structures 75 (2000) 335-345.

8. Freitas JAT, Cismaşiu C. Hybrid-Trefftz displacement element for spectral analysis of bounded and unbounded media. Int.J.Solids \& Structures 40 (2003) 671-699.

9. Sze KY, Cheung YK. A hybrid-Trefftz finite element model for Helmholtz problem. Comm. Numer.Meth.Engng. 24 (2008) 2047-2060.

10. Sze KY, Liu GH, Fan H. Four- and eight-node hybrid-Trefftz quadrilateral finite element models for Helmholtz problem. Comput.Methods Appl.Mech.Engrg. 199 (2010) 598-614.

11. Sze KY, Liu GH. Hybrid-Trefftz six-node triangular finite element models for Helmholtz problem. Computational Mechanics 46 (2010) 455-470.

12. Melenk JM, Babuška I. The partition of unity method. Int.J.Numer.Meth.Engng. 40 (1997) 727-758.

13. Mayer P, Mandel J. The finite ray element method for the Helmholtz equation of scattering: first numerical experiments. Report 111, Center of Computational Mathematics, University of Colorado at Denver, 1997.

14. Laghrouche O, Bettess P. Short wave modelling using special finite elements. J. Computational Acoustics 8 (2000) 189-210.

15. Ortiz P, Sanchez E. An improved partition of unity finite element method for diffraction problems. Inter.J.Numer.Meth.Engng. 50 (2001) 2727-2740.

16. Farhat C, Harari I, Hetmaniuk U. The discontinuous enrichment method for multiscale analysis. Computer Meth.in Appl.Mech. \& Engng. 192 (2003) 1389-1419.

17. Farhat C, Harari I, Hetmaniuk U. A discontinuous Galerkin method with Lagrange multipliers for the solution of Helmholtz problems in the mid-frequency regime. Computer Meth.in Appl.Mech. \& Engng. 192 (2003) 3195-3209.

18. Farhat C, Tezaur R, Wiedemann-Goiran. Higher-order extensions of a discontinuous Galerkin method for mid-frequency Helmholtz problems. Inter.J.Numer.Meth.Engng. 61 (2004) 1938-1956.

19. Tezaur R, Farhat C. Three-dimensional discontinuous Galerkin elements with plane waves and Lagrange multipliers for the solution of mid-frequency Helmholtz problems. Inter.J.Numer.Meth. Engng. 66 (2006) 796-815.

20. Freitas JA, Almeida JP, Pereira EMB. Non-conventional formulations for the finite element method. Comput.Mech. 23 (1999) 488-501.

21. Spilker RL, Maskeri SM, Kania E. Plane isoparametric hybrid-stress elements: invariance and optimal sampling. Int.J.Numer.Meth.Engng., 17 (1981) 1469-1496.

22. Sze KY, Chow CL, Chen WJ. On invariance of isoparametric hybrid elements. Commun.Numer. Meth.Engng. 8 (1992) 385-406.

23. Chen W, Tanaka M. A meshless, integration-free, and boundary-only RBF technique. Computers and Mathematics with Applications 43 (2002) 379-391.

24. Chen W, Fu ZJ, Jin BT. A truly boundary-only meshfree method for inhomogeneous problems based on recursive composite multiple reciprocity technique. Engineering Analysis with Boundary Elements 34 (2010) 196-205. 
25. Pian THH. State-of-the-art development of hybrid/mixed finite element method. Finite Elements in Analysis \& Design 21 (1995) 5-20.

26. Sze KY, Chan WK, Pian THH. An eight-node hybrid-stress solid-shell element for geometric nonlinear analysis of elastic shells. Int.J.Numer.Meth.Engng. 55 (2000) 853-878.

27. Cook RD, Malkus DS, Plesha ME. Concepts and Applicatins of Finite Element Analysis. $3^{\text {rd }}$ Edn., John Wiley \& Sons, 1989.

28. Liu GH. Formulation of multifield finite element models for Helmholtz problems. Ph.D. Thesis, Department of Mechanical Engineering, The University of Hong Kong. 2010.

29. Kimberling C. Triangle Centers and Central Triangles. Congressus Numerantium, 129 (1998) 1-295. 